\title{
A UNIQUENESS THEOREM FOR THERMOELASTIC SHELLS WITH GENERALIZED BOUNDARY CONDITIONS*
}

\author{
By \\ M. B. RUBIN \\ Technion, Israel Institute of Technology
}

\begin{abstract}
The uniqueness of the solution of initial, mixed boundary value problems for linear thermoelastic shells is reconsidered within the context of recent developments in the thermomechanical theory of a Cosserat surface [4]. Fairly general boundary conditions are considered which allow mechanical contact with linear elastic media and thermal radiation on the boundary curve of the Cosserat surface and on the major surfaces of the shell.
\end{abstract}

1. Introduction. Motivated by the application of shell theory to contact problems, we generalize the usual boundary conditions to include mechanical contact with linear elastic media and thermal radiation. These generalized mechanical and thermal boundary conditions are applied on the boundary curve of the shell as well as on its major surfaces. ${ }^{1}$ In this paper, the uniqueness proof is reconsidered within the context of these general boundary conditions.

By way of background, we recall that the theory of a Cosserat surface [1] has been well established as a particularly useful model of a shell-like body which, broadly speaking, is a three-dimensional body that is "thin" in one of its dimensions. Recent developments in the theory of continuum thermodynamics $[2,3]$ have provided the theoretical framework with which to develop a general thermomechanical theory of a Cosserat surface [4]. Such a general theory admits finite numbers of directors $\mathbf{d}_{N}(N=1,2, \ldots)$ and temperature fields $\theta_{N}$ to provide limited information about the variation through the thickness of the shell of the deformation and temperature field, respectively.

For less general boundary conditions than considered here, a uniqueness theorem has been proved for the linear isothermal theory of shells [1, Sec. 26] and for small motions superposed on a large deformation within the context of a thermoelastic theory of shells

\footnotetext{
* Received December 5, 1984.

${ }^{1}$ Recall [1] that the three-dimensional boundary conditions applied to the major surfaces of the shell are incorporated into the field equations and therefore are not considered to be boundary conditions in shell theory.
} 
which admits a single temperature field [5]. A uniqueness theorem for a linear thermoelastic theory of shells which admits two temperature fields and two energy equations has also been proved [6], including radiation on the major surfaces but not on the boundary curve. None of these uniqueness proofs considers mechanical contact with elastic media.

Although this latter theory [6] is not based on the recent developments for Cosserat surfaces [4], the linearized equations can be placed in a one-to-one correspondence with those of a theory which admits a single director $\mathbf{d}$ and two temperature fields $\theta, \phi$. It therefore follows that the previous uniqueness theorem [6] applies to solutions within the context of the new linearized theory [4].

In the following sections, we state sufficient conditions to prove uniqueness of the solution of the equations of the coupled thermoelastic theory ${ }^{2}$ which admits the generalized boundary conditions and which considers inhomogeneous, anisotropic elastic shells. Specifically, in Sec. 2 the basic equations of the linear theory of a Cosserat surface are recorded and in Sec. 3 the generalized boundary conditions are discussed. Finally, in Sec. 4 we state and prove the uniqueness theorem.

2. Basic equations. In this section we briefly record the relevant equations describing a thermoelastic Cosserat surface. Details of the theory of a Cosserat surface may be found in $[1,4,7]$. Here we use the notation of [7], which differs from that used in [1, 4]. For our purposes, we consider a Cosserat surface which is defined by the position vector $\mathbf{r}$, director d, and two temperature fields $\theta$ and $\phi$. A thermomechanical process of such a surface is characterized by

$$
\begin{gathered}
\mathbf{r}=\mathbf{r}\left(\theta^{\alpha}, t\right), \quad \mathbf{d}=\mathbf{d}\left(\theta^{\alpha}, t\right), \quad\left[\mathbf{a}_{1} \mathbf{a}_{2} \mathbf{d}\right]>0, \\
\theta=\theta\left(\theta^{\alpha}, t\right), \quad(\theta>0), \quad \phi=\phi\left(\theta^{\alpha}, t\right),
\end{gathered}
$$

where $\theta^{\alpha}(\alpha=1,2)$ are convected coordinates, $t$ is time, $\mathbf{a}_{\alpha}$ are tangent vectors, and $\mathbf{a}_{3}$ is the unit normal vector. The velocity $\mathbf{v}$, director velocity $\mathbf{w}$, and temperature gradients $\mathbf{g}$ and $\mathbf{g}_{1}$ are defined by ${ }^{3}$

$$
\mathbf{v}=\dot{\mathbf{r}}, \quad \mathbf{w}=\dot{\mathbf{d}}, \quad \mathbf{g}=\theta_{, \alpha} \mathbf{a}^{\alpha}, \quad \mathbf{g}_{1}=\phi_{, \alpha} \mathbf{a}^{\alpha},
$$

where a superposed dot denotes time differentiation holding $\theta^{\alpha}$ fixed, a comma denotes partial differentiation with respect to the coordinates $\theta^{\alpha}$, and $\mathbf{a}^{i}$ are the reciprocal vectors of the set $\mathbf{a}_{i}$. Furthermore, the basic kinematic quantities characterizing the reference configuration of the shell are given by

$$
\begin{aligned}
A^{1 / 2}=\left[\mathbf{A}_{1} \mathbf{A}_{2} \mathbf{A}_{3}\right] & >0, \quad A_{\alpha \beta}=\mathbf{A}_{\alpha} \cdot \mathbf{A}_{\beta}, \quad B_{\alpha \beta}=\mathbf{A}_{\alpha, \beta} \cdot \mathbf{A}_{3}, \\
D_{i} & =\mathbf{A}_{i} \cdot \mathbf{D}, \quad \Lambda_{i \alpha}=\mathbf{A}_{i} \cdot \mathbf{D}_{, \alpha},
\end{aligned}
$$

\footnotetext{
${ }^{2}$ Of course, the uniqueness theorem with its modification for the generalized boundary conditions is applicable to the purely mechanical theory as well as the purely thermal theory.

${ }^{3}$ Throughout the text we use the usual summation convention over repeated indices. Greek indices have the range $(1,2)$ and Latin indices have the range $(1,2,3)$.
} 
where $\mathbf{A}_{i}$ and $\mathbf{D}$ are the reference values of $\mathbf{a}_{i}$ and $\mathbf{d}$, respectively. For later convenience we also define the displacement $\mathbf{u}$ and director displacement $\delta$ by

$$
\mathbf{u}=\mathbf{r}-\mathbf{R}, \quad \boldsymbol{\delta}=\mathbf{d}-\mathbf{D},
$$

where $\mathbf{R}$ is the reference value of $\mathbf{r}$.

Now, the local forms of the basic equations for the linearized theory may be recorded as $[1,4,7]$ :

$$
\begin{gathered}
\lambda=\rho a^{1 / 2}=\rho_{0} A^{1 / 2}>0, \\
\lambda\left(\dot{\mathbf{v}}+y^{1} \dot{\mathbf{w}}\right)=\lambda \mathbf{f}+\left(A^{1 / 2} \mathbf{N}^{\alpha}\right)_{, \alpha}, \\
\lambda\left(y^{1} \dot{\mathbf{v}}+y^{2} \dot{\mathbf{w}}\right)=\lambda \mathbf{l}-A^{1 / 2} \mathbf{k}+\left(A^{1 / 2} \mathbf{M}^{\alpha}\right)_{, \alpha}, \\
\lambda \dot{\eta}=\lambda(s+\xi)-\left(A^{1 / 2} p^{\alpha}\right)_{, \alpha}, \\
\lambda \dot{\eta}_{1}=\lambda\left(s_{1}+\xi_{1}\right)-\left(A^{1 / 2} p_{1}^{\alpha}\right)_{, \alpha} .
\end{gathered}
$$

Equation (2.5a) represents the conservation of mass, $(2.5 \mathrm{~b})$ represents the balance of linear momentum, $(2.5 \mathrm{c})$ represents the balance of director momentum, and $(2.5 \mathrm{~d}, \mathrm{e})$ represent the balances of entropy. In Eqs. (2.5) we have introduced the following quantities: the mass density $\rho_{0}$ in the reference configuration; the present values $\rho$ and $a^{1 / 2}$ of $\rho_{0}$ and $A^{1 / 2}$, respectively; the contact force $\mathbf{N}^{\alpha}$ and contact director moment $\mathbf{M}^{\alpha}$; the specific (per unit mass) assigned force $\mathbf{f}$ and specific assigned director couple $\mathbf{~}$; the intrinsic director couple $\mathbf{k}$; the inertia coefficients $y^{1}$ and $y^{2}$; the specific entropies $\eta$ and $\eta_{1}$; the specific internal rates of production of entropy $\xi$ and $\xi_{1}$; the entropy fluxes $p^{\alpha}$ and $p_{1}^{\alpha}$; and the specific external rates of supply of entropy $s$ and $s_{1}$. Further, by referring the quantities $\mathbf{N}^{\alpha}, \mathbf{k}, \mathbf{M}^{\alpha}$ to the base vectors $\mathbf{A}_{i}$, such that ${ }^{4}$

$$
\mathbf{N}^{\alpha}=N^{i \alpha} \mathbf{A}_{i}, \quad \mathbf{k}=k^{i} \mathbf{A}_{i}, \quad \mathbf{M}^{\alpha}=M^{i \alpha} \mathbf{A}_{i},
$$

the results of angular momentum may be written in the forms

$$
\begin{gathered}
N^{\prime \beta \alpha}=N^{\alpha \beta}=N^{\beta \alpha}-k^{\alpha} D^{\beta}-M^{\alpha \sigma} \Lambda_{\cdot \sigma}^{\beta}, \\
N^{3 \alpha}=D^{3} k^{\alpha}-D^{\alpha} k^{3}+\Lambda^{3}{ }_{\cdot} M^{\alpha \sigma}-\Lambda_{{ }_{\sigma}}^{\alpha} M^{3 \sigma},
\end{gathered}
$$

where tensor quantities with superscripts are contravariant or mixed components.

Consider a shell which in its reference configuration is stress-free and has a uniform temperature $\Theta$. Then for an inhomogeneous, anisotropic elastic shell we assume constitutive equations of the forms

$$
\begin{gathered}
2 \rho_{0} \psi=2 \rho_{0} \psi_{1}-2 \beta_{0} \gamma_{3}(\theta-\Theta)-2 C_{4}^{\alpha \beta} e_{\alpha \beta}(\theta-\Theta) \\
-2 C_{5}^{\alpha \beta} \kappa_{\alpha \beta} \phi-\beta_{3}\left(\theta^{2}-2 \theta \Theta\right)-\beta_{4} \phi^{2}-2 \beta_{5} \theta, \\
2 \rho_{0} \psi_{1}=C_{1}^{\alpha \beta \gamma \delta} e_{\alpha \beta} e_{\gamma \delta}+\alpha_{4}\left(\gamma_{3}\right)^{2}+C_{2}^{\alpha \beta \gamma \delta} \kappa_{\alpha \beta} \kappa_{\gamma \delta} \\
+C_{1}^{\alpha \beta} \gamma_{\alpha} \gamma_{\beta}+C_{2}^{\alpha \beta} \kappa_{3 \alpha} \kappa_{3 \beta}+2 C_{3}^{\alpha \beta} e_{\alpha \beta} \gamma_{3}, \\
p^{\alpha}=-C_{6}^{\alpha \beta} \theta_{, \beta}, \quad p_{1}^{\alpha}=-C_{7}^{\alpha \beta} \phi_{, \beta},
\end{gathered}
$$

\footnotetext{
${ }^{4}$ This notation is consistent with [7] but differs from that used in $[1,4]$.
} 


$$
\begin{gathered}
\rho_{0} \theta \xi=C_{6}^{\alpha \beta} \theta_{, \alpha} \theta_{, \beta}+C_{7}^{\alpha \beta} \phi_{, \alpha} \phi_{, \beta}+b_{2} \phi^{2}, \\
\rho_{0} \xi_{1}=\rho_{0} \bar{\xi}_{1}=-b_{2} \phi,
\end{gathered}
$$

where $e_{\alpha \beta}, \gamma_{i}, \kappa_{i \alpha}$ are strain measures [1], $\psi$ is the Helmholtz free energy, $\bar{\xi}_{1}$ is a specific internal production of entropy, the quantities $C_{N}^{\alpha \beta \gamma \delta}(N=1,2), C_{N}^{\alpha \beta}(N=1,2, \ldots, 7), \alpha_{4}$, $\beta_{0}, \beta_{3}, \beta_{4}, \beta_{5}, b_{2}$ are functions of the variables

$$
V=\left\{\theta^{\alpha}, A_{\alpha \beta}, B_{\alpha \beta}, D_{i}, \Lambda_{i \alpha}, \Theta\right\},
$$

and the tensors $C_{N}^{\alpha \beta \gamma \delta}$ and $C_{N}^{\alpha \beta}$ have appropriate symmetries.

The forms of the constitutive equations (2.8) are chosen to be similar to those used in the linearized theory [4], but with appropriate generalizations to allow for anisotropic, inhomogeneous response and to satisfy the reduced energy equation without approximation. In particular, we note from (2.8) that $\xi$ is of higher order and therefore may be neglected in Eq. (2.5d). Now for an elastic shell we may deduce the additional results [4]:

$$
\begin{gathered}
N^{\alpha \beta}=C_{1}^{\alpha \beta \gamma \delta} e_{\gamma \delta}+C_{3}^{\alpha \beta} \gamma_{3}-C_{4}^{\alpha \beta}(\theta-\Theta), \\
k^{\alpha}=C_{1}^{\alpha \beta} \gamma_{\beta}, \quad k^{3}=\alpha_{4} \gamma_{3}+C_{3}^{\alpha \beta} e_{\alpha \beta}-\beta_{0}(\theta-\Theta), \\
M^{\alpha \beta}=C_{2}^{\alpha \beta \gamma \delta} \kappa_{\gamma \delta}-C_{5}^{\alpha \beta} \phi, \quad M^{3 \alpha}=C_{2}^{\alpha \beta} \kappa_{3 \beta}, \\
\rho_{0} \eta=\beta_{0} \gamma_{3}+C_{4}^{\alpha \beta} e_{\alpha \beta}+\beta_{3}(\theta-\Theta)+\beta_{5}, \quad \rho_{0} \eta_{1}=C_{5}^{\alpha \beta} \kappa_{\alpha \beta}+\beta_{4} \phi, \\
2 \rho_{0} \varepsilon=2 \rho_{0} \psi_{1}+2 \Theta \beta_{0} \gamma_{3}+2 \Theta C_{4}^{\alpha \beta} e_{\alpha \beta}+\beta_{3} \theta^{2}+\beta_{4} \phi^{2},
\end{gathered}
$$

where $\varepsilon=\psi+\theta \eta+\theta \eta_{1}$ is the specific internal energy.

Finally, we recall [4] that for such an elastic shell the only nontrivial statements of the second law of thermodynamics take the forms

$$
\begin{gathered}
\rho_{0} \phi \bar{\xi}_{1}+\mathbf{p} \cdot \mathbf{g}+\mathbf{p}_{1} \cdot \mathbf{g}_{1} \leqslant 0, \\
\theta(t)-\theta_{1}>0 \quad \text { whenever } \varepsilon(t)-\varepsilon_{1}>0 .
\end{gathered}
$$

The statement (2.11a) corresponds to the classical heat conduction inequality and is assumed valid for all equilibrium displacement and temperature fields. Further, the statement (2.11b) is assumed valid when the Cosserat surface is at rest and the three-dimensional temperature field is spatially uniform so that $\theta=\theta(t), \phi=0$. In $(2.11 \mathrm{~b}), \varepsilon_{1}$ and $\theta_{1}$ correspond to the internal energy and uniform temperature of the shell during some period of time up to $t_{1}$ when the shell has been at rest and in thermal equilibrium.

3. Boundary and initial conditions. This theory, which is developed by a direct approach, may be brought into a one-to-one correspondence with the three-dimensional theory by assuming that the position vector $\mathbf{p}^{*}$ of a point in the shell and the temperature field $\theta^{*}$ admit the representations

$$
\begin{aligned}
\mathbf{p}^{*}=\mathbf{p}^{*}\left(\theta^{\alpha}, \theta^{3}, t\right) & =\mathbf{r}\left(\theta^{\alpha}, t\right)+\theta^{3} \mathbf{d}\left(\theta^{\alpha}, t\right), \\
\theta^{*} & =\theta^{*}\left(\theta^{\alpha}, \theta^{3}, t\right)=\theta\left(\theta^{\alpha}, t\right)+\theta^{3} \phi\left(\theta^{\alpha}, t\right),
\end{aligned}
$$

where $\theta^{3}$ is a coordinate through the thickness of the shell. Without loss of generality, we may define the top surface $\partial P^{+}$of the shell by $\theta^{3}=h / 2$, where $h$ is a constant having the dimensions of length, and write the displacement $\mathbf{u}^{+}$and temperature difference $\left(\theta^{+}-\Theta\right)$ 
on $\partial P^{+}$as

$$
\mathbf{u}^{+}=\mathbf{u}+\frac{h}{2} \delta, \quad\left(\theta^{+}-\Theta\right)=(\theta-\Theta)+\frac{h}{2} \phi .
$$

Similarly, we may define the bottom surface $\partial P^{-}$of the shell by $\theta^{3}=-h / 2$ and write the displacement $\mathbf{u}^{-}$and temperature difference $\left(\theta^{-}-\Theta\right)$ on $\partial P^{-}$by

$$
\mathbf{u}^{-}=\mathbf{u}-\frac{h}{2} \delta, \quad\left(\theta^{-}-\Theta\right)=(\theta-\Theta)-\frac{h}{2} \phi .
$$

With the help of (2.7) and the constitutive equations $(2.8 \mathrm{c}, \mathrm{d}, \mathrm{f}),(2.10 \mathrm{a}-\mathrm{g})$, and appropriate strain-displacement relations [1], Eqs. (2.5), with $\xi=0$, represent a system of equations to determine the unknowns $\mathbf{u}, \boldsymbol{\delta}, \boldsymbol{\theta}, \boldsymbol{\phi}$. These equations must be solved subject to certain initial and boundary conditions. Let $P$, bounded by the closed curve $\partial P$, denote the region occupied by an arbitrary material portion of the Cosserat surface in the present configuration. Then the initial conditions may be defined at each point of $P$ by specifying

$$
\begin{gathered}
\mathbf{u}=\mathbf{u}_{0}\left(\theta^{\alpha}\right), \quad \delta=\delta_{0}\left(\theta^{\alpha}\right), \quad \theta=\theta_{0}\left(\theta^{\alpha}\right), \quad \phi=\phi_{0}\left(\theta^{\alpha}\right), \quad(3.4 \mathrm{a}, \mathrm{b}, \mathrm{c}, \mathrm{d}) \\
\dot{\mathbf{u}}=\mathbf{v}_{0}\left(\theta^{\alpha}\right), \quad \delta=\mathbf{w}_{0}\left(\theta^{\alpha}\right), \quad \text { at } t=t_{0}, \quad(3.4 \mathrm{e}, \mathrm{f})
\end{gathered}
$$

where $\mathbf{u}_{0}, \mathbf{v}_{0}, \boldsymbol{\delta}_{0}, \mathbf{w}_{0}, \theta_{0}, \phi_{0}$ are specified functions of $\theta^{\alpha}$ only. Mixed ${ }^{5}$ boundary conditions which include mechanical contact with a linear elastic medium or thermal radiation may be written at each point ${ }^{6} s$ of the boundary curve $\partial P$ in the generalized forms ${ }^{7}$ :

$$
\begin{array}{r}
\mathbf{n}+\mathbf{B}(s) \mathbf{u}+\mathbf{C}(s, t)=0, \quad \mathbf{m}+\mathbf{B}_{1}(s) \delta+\mathbf{C}_{1}(s, t)=0, \quad(3.5 \mathrm{a}, \mathrm{b}) \\
k-B(s, t)(\theta-\Theta)+C(s, t)=0, \quad k_{1}-B_{1}(s, t) \phi+C_{1}(s, t)=0, \quad \text { for } t \in\left[t_{0}, \infty\right),
\end{array}
$$

where

$$
\mathbf{n}=\mathbf{N}^{\alpha} \nu_{\alpha}, \quad \mathbf{m}=\mathbf{M}^{\alpha} \nu_{\alpha}, \quad k=p^{\alpha} \nu_{\alpha}, \quad k_{1}=p_{1}^{\alpha} \nu_{\alpha}
$$

and where $\nu_{\alpha}$ are components of the unit outward normal vector of the curve $\partial P$. In (3.5) $\mathbf{B}$ and $\mathbf{B}_{1}$ are assumed to be continuous, symmetric, three-dimensional, second-order tensor functions of $s$ and are independent of time; $B$ and $B_{1}$ are continuous scalar functions of $(s, t) ; \mathbf{C}$ and $\mathbf{C}_{1}$ are continuous vector functions of $(s, t)$; and $C$ and $C_{1}$ are continuous scalar functions of $(s, t)$. For later convenience, we define the scalars

$$
J_{1}=\frac{1}{2} \mathbf{u} \cdot \mathbf{B u}, \quad J_{2}=\frac{1}{2} \delta \cdot \mathbf{B}_{1} \delta .
$$

A boundary condition on $\mathbf{n}$ alone can be obtained trivially from (3.5a) by setting $\mathbf{B}=0$, and a boundary condition on $\mathbf{u}$ alone can be obtained from (3.5a) by setting the tensor $\mathbf{B}$ equal to a scalar $b$ times the identity tensor $\mathbf{I}(\mathbf{B}=b \mathbf{I})$ and then taking the limit as $b$ approaches infinity.

\footnotetext{
${ }^{5}$ Mixed-mixed boundary conditions may be specified but are not considered explictly.

${ }^{6}$ The temporary use of the symbol $s$ for a point on $\partial P$ should not be confused with the use of the same symbol elsewhere for the external entropy supply.

${ }^{7} \mathrm{~A}$ condition of the type (3.5c) has previously been considered for the three-dimensional theory [8, Sec. 5.7].
} 
Recall from [4] that the assigned fields $\mathbf{f}, \mathbf{I}, \mathbf{s}$, and $s_{1}$ include contributions from both the effects of three-dimensional body force and external entropy supply as well as from the effects of surface tractions and entropy flux on the major surfaces of the shell. In view of the specification $\theta^{3}= \pm h / 2$ defining the major surfaces, we may write these assigned fields in the forms

$$
\begin{gathered}
\lambda \mathbf{f}=\lambda \overline{\mathbf{f}}+A^{1 / 2}\left(b^{+} \mathbf{t}^{+}+b^{-} \mathbf{t}^{-}\right), \\
\lambda \mathbf{I}=\lambda \overline{\mathbf{I}}+A^{1 / 2}\left(\frac{h}{2}\right)\left(b^{+} \mathbf{t}^{+}-b^{-} \mathbf{t}^{-}\right), \\
\lambda s=\lambda \bar{s}-A^{1 / 2}\left(b^{+} k^{+}+b^{-} k^{-}\right), \\
\lambda s_{1}=\lambda \bar{s}_{1}-A^{1 / 2}\left(\frac{h}{2}\right)\left(b^{+} k^{+}-b^{-} k^{-}\right),
\end{gathered}
$$

where $\overline{\mathbf{f}}, \overline{\mathbf{l}}, \overline{\mathbf{s}}, \bar{s}_{1}$ are considered to be specified assigned fields associated with the three-dimensional body force and external supply of entropy, $b^{+}$and $b^{-}$are positive scalar functions of the variables (2.9) and are independent of time, $\mathbf{t}^{+}$is the traction vector and $k^{+}$is the entropy flux on the major surface $\partial P^{+}$, and $\mathbf{t}^{-}$is the traction vector and $k^{-}$ is the entropy flux on the major surface $\partial P^{-}$. To allow mechanical contact of the major surfaces with an elastic medium and to allow thermal radiation, we assume that at each point of ${ }^{8} \partial P^{+}$,

$$
\mathbf{t}^{+}+\mathbf{B}^{+} \mathbf{u}^{+}+\mathbf{C}^{+}=0, \quad k^{+}-B^{+}\left(\theta^{+}-\Theta\right)+C^{+}=0,
$$

and at each point of $\partial P^{-}$,

$$
\mathbf{t}^{-}+\mathbf{B}^{-} \mathbf{u}^{-}+\mathbf{C}^{-}=0, \quad k^{-}-B^{-}\left(\theta^{-}-\Theta\right)+C^{-}=0,
$$

where $\mathbf{B}^{+}$and $\mathbf{B}^{-}$are continuous, symmetric, three-dimensional, second-order tensor functions of the variables $V$ in (2.9) and are independent of time; $\mathbf{C}^{+}$and $\mathbf{C}^{-}$are continuous vector functions of the variables $(V, t)$; and $B^{+}, B^{-}, C^{+}, C^{-}$are continuous scalar functions of the variables $(V, t)$. For later convenience, we define the scalars

$$
J_{3}=\frac{1}{2} \mathbf{u}^{+} \cdot \mathbf{B}^{+} \mathbf{u}^{+}, \quad J_{4}=\frac{1}{2} \mathbf{u}^{-} \cdot \mathbf{B}^{-} \mathbf{u}^{-} .
$$

Depending on the nature of the problem to be considered, the quantities $\mathbf{t}^{+}, \mathbf{t}^{-}, k^{+}, k^{-}$, $\mathbf{u}^{+}, \mathbf{u}^{-}, \boldsymbol{\theta}^{+}, \theta^{-}$are either specified or to be determined by the field equations. For example, if we were to consider a slightly more general boundary condition of the type (3.9a) which specified the tangential components of $\mathbf{t}^{+}$and the normal component of $\mathbf{u}^{+}$, then the normal component of $\mathbf{t}^{+}$and the tangential components of $\mathbf{u}^{+}$would be determined by the field equations. This is similar to the use of such quantities in the theory of laminated composite plates [9].

4. A uniqueness theorem. We now state the following uniqueness theorem: Let $\mathbf{u}, \boldsymbol{\delta}, \theta, \phi$ be displacements and temperature fields which satisfy the above mentioned linear field equations, constitutive equations, and statements of the second law of thermodynamics

\footnotetext{
${ }^{8}$ Note that the range of the convected coordinates $\theta^{\alpha}$ on the major surfaces $\partial P^{+}$and $\partial P^{-}$is the same as that on the reference surface $P$ of the Cosserat surface.
} 
(2.11) on $P \times\left[t_{0}, \infty\right)$, and satisfy the initial conditions on $P$ at $t=t_{0}$, the boundary conditions on $\partial P \times\left[t_{0}, \infty\right)$, conditions of the type (3.9) on $\partial P^{+} \times\left[t_{0}, \infty\right)$ and (3.10) on $\partial P^{-} \times\left[t_{0}, \infty\right)$, for prescribed values of the assigned force $\overline{\mathbf{f}}$, director couple $\mathbf{i}$, and external supplies of entropy $\bar{s}$ and $\bar{s}_{1}$. Then, provided the specific kinetic energy $K$ defined by

$$
K=K(\mathbf{v}, \mathbf{w})=\frac{1}{2}\left(\mathbf{v} \cdot \mathbf{v}+2 y^{1} \mathbf{v} \cdot \mathbf{w}+y^{2} \mathbf{w} \cdot \mathbf{w}\right)
$$

is positive definite, the specific heats $\beta_{3}$ and $\beta_{4}$ in (2.8a) are positive scalars, the portion of the Helmholtz free energy $\psi_{1}$ in (2.8b) is positive semidefinite, and the scalars $J_{1}, J_{2}, J_{3}$, $J_{4}, B, B_{1}, B^{+}, B^{-}$in (3.5), (3.7), (3.9)-(3.11) are positive semidefinite, there exists at most one set of functions $\mathbf{u}, \delta, \theta, \phi$ which satisfy the strain-displacement relations, the field equations (2.5) [with $\xi=0$ in (2.5d)] and (2.7), the constitutive equations (2.8) and (2.10), the restriction (2.11a), initial conditions (3.4), boundary conditions (3.5), and conditions (3.9) and (3.10), and are of class $C^{1}$ on $\partial P \times\left[t_{0}, \infty\right)$ and are of class $C^{2}$ on $P \times\left[t_{0}, \infty\right)$. For convenience, the restrictions stated above may be written in the mathematical forms ${ }^{9}$

$$
\begin{gathered}
y^{2}-\left(y^{1}\right)^{2}>0, \quad \beta_{3}>0, \quad \beta_{4}>0, \quad \psi_{1} \geqslant 0, \\
J_{1} \geqslant 0, \quad J_{2} \geqslant 0, \quad J_{3} \geqslant 0, \quad J_{4} \geqslant 0, \\
B \geqslant 0, \quad B_{1} \geqslant 0, \quad B^{+} \geqslant 0, \quad B^{-} \geqslant 0 .
\end{gathered}
$$

Apart from the discussion of the generalized boundary conditions, our method of proof is nearly identical to that used in [6]. Specifically, we assume the existence of two different solutions of the initial, mixed boundary-value problem stated above, form the difference solution, and use a consequence of the field equations to prove that the difference solution is the null solution. Let us denote a typical variable of the difference solution by $\hat{U}$. Then it follows that the difference solution satisfies the field equations

$$
\begin{gathered}
\lambda\left(\dot{\hat{\mathbf{v}}}+y^{1} \dot{\hat{\mathbf{w}}}\right)=A^{1 / 2}\left(b^{+} \mathbf{t}^{+}+b^{-} \mathbf{t}^{-}\right)+\left(A^{1 / 2} \hat{\mathbf{N}}^{\alpha}\right)_{, \alpha}, \\
\lambda\left(y^{1} \dot{\hat{\mathbf{v}}}+y^{2} \dot{\hat{\mathbf{w}}}\right)=A^{1 / 2}\left(\frac{h}{2}\right)\left(b^{+} \hat{\mathbf{t}}^{+}-b^{-} \mathbf{t}^{-}\right)-A^{1 / 2} \hat{\mathbf{k}}+\left(A^{1 / 2} \hat{\mathbf{M}}^{\alpha}\right)_{, \alpha} \\
\lambda \dot{\hat{\eta}}=-A^{1 / 2}\left(b^{+} \hat{k}^{+}+b^{-} \hat{k}^{-}\right)-\left(A^{1 / 2} \hat{p}^{\alpha}\right)_{, \alpha}, \\
\lambda \dot{\hat{\eta}}_{1}=\lambda \hat{\xi}_{1}-A^{1 / 2}\left(\frac{h}{2}\right)\left(b^{+} \hat{k}^{+}-b^{-} \hat{k}^{-}\right)-\left(A^{1 / 2} \hat{p}_{1}^{\alpha}\right)_{, \alpha},
\end{gathered}
$$

on $P \times\left[t_{0}, \infty\right)$, the restriction

$$
\rho_{0} \hat{\phi} \hat{\xi}+\hat{\mathbf{p}} \cdot \hat{\mathbf{g}}+\hat{\mathbf{p}}_{1} \cdot \hat{\mathbf{g}}_{1} \leqslant 0
$$

on $P \times\left[t_{0}, \infty\right)$, the initial conditions

$$
\hat{\mathbf{u}}=0, \quad \hat{\boldsymbol{\delta}}=0, \quad \hat{\boldsymbol{\theta}}=0, \quad \hat{\boldsymbol{\phi}}=0, \quad \dot{\hat{\mathbf{u}}}=0, \quad \dot{\hat{\boldsymbol{\delta}}}=0,
$$

on $P$ at $t=t_{0}$, the boundary conditions

$$
\begin{array}{ll}
\hat{\mathbf{n}}+\mathbf{B} \hat{\mathbf{u}}=0, & \hat{\mathbf{m}}+\mathbf{B}_{1} \hat{\boldsymbol{\delta}}=0 \\
\hat{k}-B \hat{\boldsymbol{\theta}}=0, & \hat{k}_{1}-B_{1} \hat{\boldsymbol{\phi}}=0
\end{array}
$$

\footnotetext{
${ }^{9}$ The restriction $(4.2 b)$ is consistent with the condition $(2.11 b)$.
} 
on $\partial P \times\left[t_{0}, \infty\right)$, the conditions

$$
\mathbf{t}^{+}+\mathbf{B}^{+} \hat{\mathbf{u}}^{+}=0, \quad \hat{k}^{+}-B^{+} \hat{\boldsymbol{\theta}}^{+}=0,
$$

on $\partial P^{+} \times\left[t_{0}, \infty\right)$, and the conditions

$$
\mathbf{t}^{-}+\mathbf{B}^{-} \hat{\mathbf{u}}^{-}=0, \quad \hat{k}^{-}-B^{-} \hat{\boldsymbol{\theta}}^{-}=0,
$$

on $\partial P^{-} \times\left[t_{0}, \infty\right)$.

Multiplying (4.3c) by $\hat{\theta},(4.3 \mathrm{~d})$ by $\hat{\phi}$, adding the results together, integrating over the region $P$, using the divergence theorem and the conditions $(4.6 \mathrm{c}, \mathrm{d}),(4.7 \mathrm{~b})$, and $(4.8 \mathrm{~b})$, we obtain

$$
\begin{aligned}
& \int_{P} \rho_{0}\left(\hat{\theta} \dot{\hat{\eta}}+\hat{\phi} \dot{\hat{\eta}}_{1}\right) d \sigma=\int_{P}\left(\rho_{0} \hat{\phi} \hat{\xi}_{1}+\hat{\mathbf{p}} \cdot \hat{\mathbf{g}}+\hat{\mathbf{p}}_{1} \cdot \hat{\mathbf{g}}_{1}\right) d \sigma \\
& -\int_{P}\left[b^{+} B^{+}\left(\hat{\theta}^{+}\right)^{2}+b^{-} B^{-}\left(\hat{\theta}^{-}\right)^{2}\right] d \sigma-\int_{\partial P}\left(B \hat{\theta}^{2}+B_{1} \hat{\phi}^{2}\right) d s,
\end{aligned}
$$

where $d \sigma$ is the area element on $P$ and $d s$ is the arc length on $\partial P$. Taking the inner product of (4.3a) with $\hat{\mathbf{v}},(4.3 \mathrm{~b})$ with $\hat{\mathbf{w}}$, adding the results together, integrating over the region $P$, using the divergence theorem and the conditions (4.6a,b), $(4.7 \mathrm{a})$, and $(4.8 \mathrm{a})$, we deduce the expression

$$
\dot{E}=\int_{P} \rho_{0}\left(\hat{\theta} \dot{\hat{\eta}}+\hat{\phi} \dot{\hat{\eta}}_{1}\right) d \sigma
$$

where

$$
\begin{gathered}
E=\int_{P}\left[\rho_{0}\left(\hat{\psi}_{1}+\hat{K}\right)+\frac{1}{2} \beta_{3} \hat{\theta}^{2}+\frac{1}{2} \beta_{4} \hat{\phi}^{2}+b^{+} \hat{J}_{3}+b^{-} \hat{J}_{4}\right] d \sigma+\int_{\partial P}\left(\hat{J}_{1}+\hat{J}_{2}\right) d s,(4.11 \mathrm{a}) \\
\hat{\psi}_{1}=\psi_{1}\left(\hat{e}_{\alpha \beta}, \hat{\gamma}_{i}, \hat{\kappa}_{i \alpha}\right), \quad \hat{K}=K(\hat{\mathbf{v}}, \hat{\mathbf{w}}), \quad \hat{J}_{1}=J_{1}(\hat{\mathbf{u}}), \\
\hat{J}_{2}=J_{2}(\hat{\boldsymbol{\delta}}), \quad \hat{J}_{3}=J_{3}\left(\hat{u}^{+}\right), \quad \hat{J}_{4}=J_{4}\left(\hat{u}^{-}\right),
\end{gathered}
$$

and where the functions $\psi_{1}, K, J_{1}-J_{4}$, are defined by (2.8b), (4.1), (3.7), and (3.11), respectively. Now from (2.8f), (4.9)-(4.11), and the restrictions (2.11b) and (4.2), we realize that $E \geqslant 0$ and $\dot{E} \leqslant 0$. Using the initial conditions (4.5), we obtain the result that $E=0$ for all time $t \in\left[t_{0}, \infty\right)$ and therefore

$$
\hat{\mathbf{v}}=0, \quad \hat{\mathbf{w}}=0, \quad \hat{\boldsymbol{\theta}}=0, \quad \hat{\boldsymbol{\phi}}=0 .
$$

Integrating (4.12a,b) and using the initial conditions (4.5), we have

$$
\hat{\mathbf{u}}=0, \quad \hat{\boldsymbol{\delta}}=0 .
$$

Using $(4.12 c, d),(4.13 a, b)$, and the expressions (3.2) and (3.3), we deduce the additional results

$$
\hat{\mathbf{u}}^{+}=0, \quad \hat{\mathbf{u}}^{-}=0, \quad \hat{\boldsymbol{\theta}}^{+}=0, \quad \hat{\boldsymbol{\theta}}^{-}=0 .
$$

Finally, substituting (4.12c,d), (4.13), and (4.14) into conditions (4.6)-(4.8), we conclude that

$$
\begin{aligned}
& \hat{\mathbf{n}}=0, \quad \hat{\mathbf{m}}=0, \quad \mathbf{t}^{+}=0, \quad \mathbf{t}^{-}=0, \\
& \hat{k}=0, \quad \hat{k}_{1}=0, \quad \hat{k}^{+}=0, \quad \hat{k}^{-}=0,
\end{aligned}
$$

which completes the proof. 
To prove uniqueness for static problems of thermoelastic shells, we need slightly stronger conditions than (2.11a) and (4.2d). Specifically, we retain the restrictions (4.2e-1) and assume that $\psi_{1}$ is positive definite and that the expression on the left-hand side of (2.11a) is negative definite so that

$$
\psi_{1}>0, \quad \rho_{0} \phi \bar{\xi}_{1}+\mathbf{p} \cdot \mathbf{g}+\mathbf{p}_{1} \cdot \mathbf{g}_{1}<0
$$

where $\psi_{1}$ in (4.16a) vanishes only when the mechanical fields $e_{\alpha \beta}, \gamma_{i}, \kappa_{i \alpha}$ vanish and (4.16b) vanishes only when the thermal fields $\theta_{, \alpha}, \phi, \phi_{, \alpha}$ vanish. Furthermore, we require the temperature $\theta$ to be specified at at least one point on the boundary of the shell. This can be done by specifying $\theta$ on $\partial P, \theta^{+}$on $\partial P^{+}$, or $\theta^{-}$on $\partial P^{-}$.

Now, for static problems the thermal equations $(4.3 \mathrm{c}, \mathrm{d})$ are uncoupled from the mechanical equations $(4.3 \mathrm{a}, \mathrm{b})$ and the expression (4.9) can be derived with the left-hand side vanishing. It follows that ${ }^{10}$

$$
\rho_{0} \hat{\phi} \hat{\xi}_{1}+\hat{\mathbf{p}} \cdot \hat{\mathbf{g}}+\hat{\mathbf{p}}_{1} \cdot \hat{\mathbf{g}}_{1}=0
$$

from which we conclude that

$$
\hat{\mathbf{g}}=0, \quad \hat{\boldsymbol{\phi}}=0 .
$$

Integrating (4.18a) and using the specification of $\theta$ at a point of the boundary of the shell as well as expressions (3.2b), (3.3b), and (4.18b), we have

$$
\hat{\boldsymbol{\theta}}=0, \quad \hat{\boldsymbol{\theta}}^{+}=0, \quad \hat{\boldsymbol{\theta}}^{-}=0 .
$$

Substituting (4.18b) and (4.19) into conditions (4.6c,d), (4.7b), and (4.8b), we deduce that

$$
\hat{k}=0, \quad \hat{k}_{1}=0, \quad \hat{k}^{+}=0, \quad \hat{k}^{-}=0,
$$

which completes the proof for the thermal fields. In view of the results (4.18b) and (4.19), we may take the inner product of the equilibrium forms of Eqs. (4.3a) and (4.3b) with $\hat{\mathbf{u}}$ and $\hat{\delta}$, respectively, and derive the expression

$$
\int_{P}\left(\rho_{0} \hat{\psi}_{1}+b^{+} \hat{J}_{3}+b^{-} \hat{J}_{4}\right) d \sigma+\int_{\partial P}\left(\hat{J}_{1}+\hat{J}_{2}\right) d s=0 .
$$

It follows that $\hat{\psi}_{1}=0$ so that

$$
\hat{e}_{\alpha \beta}=0, \quad \hat{\gamma}_{i}=0, \quad \hat{\kappa}_{i \alpha}=0 .
$$

Hence, the displacements are unique to within a linear superposed rigid body displacement. If this arbitrariness is removed, then the displacements will be unique. Thus with the help of (3.2) and (3.3) we have

$$
\hat{\mathbf{u}}=0, \quad \hat{\boldsymbol{\delta}}=0, \quad \hat{\mathbf{u}}^{+}=0, \quad \hat{\mathbf{u}}^{-}=0,
$$

and from conditions (4.6a,b), (4.7a), and (4.8a), we can conclude that

$$
\hat{\mathbf{n}}=0, \quad \hat{\mathbf{m}}=0, \quad \hat{\mathbf{t}}^{+}=0, \quad \mathbf{t}^{-}=0,
$$

which completes the proof for the mechanical fields.

\footnotetext{
${ }^{10}$ Recall from (2.8f) that $\bar{\xi}_{1}=\xi_{1}$.
} 
Acknowledgment. This research was supported by the U.S. Air Force Office of Scientific Research under contract F49620-84K-0001 with SRI International, Menlo Park, California.

\section{REFERENCES}

[1] P. M. Naghdi, The theory of shells and plates, in S. Flügge's Handbuch der Physik, vol. VIa/2 (C. Truesdell, ed.), pp. 425-640, Springer-Verlag, Berlin (1972)

[2] A. E. Green and P. M. Naghdi, On thermodynamics and the nature of the second law, Proc. Roy. Soc. London Ser. A 357, 253-270 (1977)

[3] A. E. Green and P. M. Naghdi, The second law of thermodynamics and cyclic processes, J. Appl. Mech. 45. 487-492 (1978)

[4] A. E. Green and P. M. Naghdi, On thermal effects in the theory of shells, Proc. Roy. Soc. London Ser. A 365 , $161-190$ (1979)

[5] P. M. Naghdi and J. A. Trapp, A uniqueness theorem in the theory of Cosserat surfaces, J. Elast. 2, 9-20 (1972)

[6] A. E. Green and P. M. Naghdi, On uniqueness in the linear theory of elastic shells and plates, J. Méc. 10, no. 2, 251-261 (1971)

[7] P. M. Naghdi, Finite deformation of elastic rods and shells, Proc. IUTAM Symp. Finite Elasticity, Bethlehem, Pa., 1980 (D. E. Carlson and R. T. Shield, eds.), pp. 47-103, Martinus Nijhoff, The Hague (1982)

[8] B. A. Boley and J. H. Weiner, Theory of thermal stresses, Wiley, New York (1960)

[9] A. E. Green and P. M. Naghdi, A theory of laminated composite plates, IMA J. Appl. Math. 29, 1-23 (1982) 